\title{
Impact of obinutuzumab alone and in combination for follicular lymphoma
}

This article was published in the following Dove Press journal:

Blood and Lymphatic Cancer:Targets and Therapy

19 October 2017

Number of times this article has been viewed

\author{
Maryam Sarraf Yazdy \\ Bruce D Cheson \\ Division of Hematology-Oncology, \\ Georgetown University Hospital, \\ Lombardi Comprehensive Cancer \\ Center, Washington, DC, USA
}

\begin{abstract}
Although rituximab-based chemoimmunotherapy prolongs the survival of patients with follicular lymphoma (FL), this disease is considered incurable in most patients. Thus, new therapies are needed not only for those in the relapsed/refractory setting, but also for initial treatment. Obinutuzumab (G, GA101) is a third-generation, fully humanized type II glycoengineered, anti-CD20 monoclonal antibody that results in increased direct cell death and antibody-dependent, cell-mediated cytotoxicity/phagocytosis compared to rituximab. Obinutuzumab has significant antitumor activity when used alone or in combinations in untreated or relapsed refractory FL patients. Studies have demonstrated its ability to prolong progression-free survival and, in some cases, overall survival, and to eliminate minimal residual disease. Several ongoing trials are investigating combinations with chemotherapy, immunomodulators, targeted drugs, and immunotherapy agents. $G$ is generally well tolerated, with associated adverse effects including infusion-related reactions, neutropenia, thrombocytopenia, and reactivation of hepatitis $B$ virus. Future studies with this antibody should focus on identifying predictive markers and developing chemotherapy-free combinations that will improve the outcome of patients with FL.
\end{abstract}

Keywords: obinutuzumab, follicular lymphoma, MRD, monoclonal antibody

\section{Introduction}

The improved survival of patients with follicular lymphoma (FL) has resulted from the incorporation of rituximab (Genentech, Inc., South San Francisco, CA, USA) into chemoimmunotherapy regimens. However, not all patients benefit from such approaches. Recent studies suggest that patients who fail to maintain a remission of 1 year, 2 years, or 30 months have a significantly inferior outcome to those who do not experience an event. ${ }^{1-3}$ Twenty percent of patients with FL relapse within 2 years of treatment have an inferior 5-year overall survival (OS) of 50\% compared to $90 \%$ for patients who did not relapse early. Nevertheless, the disease is considered incurable in most patients. ${ }^{4-7}$ Patients typically experience multiple relapses and eventually develop resistance to treatment, often including rituximab. ${ }^{8}$ Numerous mechanisms of rituximab resistance have been hypothesized, including decreased cellular penetration, impaired rituximab binding (via FcyRIII polymorphisms), downregulation of CD20, increased rituximab metabolism, resistance of tumor cells to rituximab-effector mechanisms, impaired immune effector cell recruitment or function, and complement depletion and downregulation of proapoptotic proteins. ${ }^{9-12}$

The poor outcome of rituximab-refractory patients has led to a search for more effective agents to improve patient outcome, including monoclonal antibodies (mAbs).
Correspondence: Bruce D Cheson Lombardi Comprehensive Cancer Center, MedStar Georgetown University Hospital, 3800 Reservoir Road NW, Washington, DC 20007, USA

Tel +I 2024447932

$\mathrm{Fax}+\mathrm{I} 202444$ I229

Email bdc@georgetown.edu 
For an antibody to establish a place in current strategies, it should either be more active than rituximab, be effective in the setting of rituximab resistance, or prolong survival when given as maintenance.

MAbs against CD20 are divided into two classes: type I (i.e., rituximab, ofatumumab [Novartis Pharmaceuticals Corporation, East Hanover, NJ, USA], veltuzumab [Immunomedics, Inc., Morris Plains, NJ, USA], and ublituximab [TG Therapeutics, Inc., New York, NY, USA]) and type II (i.e., obinutuzumab [Genentech, Inc.] and tositumomab [GlaxoSmithKline LLC., Wilmington, DE, USA]). ${ }^{13}$ Upon binding, type I antibodies induce a translocation of CD20 into large lipid rafts within the plasma membrane, which does not occur in type II antibodies. ${ }^{14}$ This event boosts complement activation and results in high complementdependent cytotoxicity (CDC) in type I antibodies, whereas type II antibodies have low CDC induction. ${ }^{15}$ Internalization of CD20 antibody complex after binding of rituximab results in decreased effector cell recruitment and antibody half-life whereas type II mAbs demonstrate minimal CD20 internalization. ${ }^{16}$ Only half as many type II antibodies bind per B-cell as type I mAbs. ${ }^{13}$ The biological implications of this finding are unknown.

Multiple other second-generation anti-CD20-directed mAbs have been developed. Ofatumumab is a type I mAb with greater $\mathrm{CDC}$ compared with rituximab, and it detaches more slowly from CD20. ${ }^{17}$ It was first approved in patients with chronic lymphocytic leukemia (CLL) who were refractory to alemtuzumab and fludarabine (Ben Venue Laboratories, Bedford, OH, USA). ${ }^{18}$ Ofatumumab demonstrated minimal single-agent activity in rituximab-refractory FL. ${ }^{19}$ In a randomized, multicenter Phase II study of ofatumumab, Rosenbaum et al randomized 51 patients with untreated FL to either $500 \mathrm{mg}(\mathrm{n}=15)$ or $1000 \mathrm{mg}(\mathrm{n}=36)$ four weekly doses followed by an extended induction phase with four additional doses of ofatumumab every 8 weeks. ${ }^{20}$ The overall response rate (ORR) was $86.7 \%$ and $60 \%$ in 1000 and $500 \mathrm{mg}$ arms, respectively, suggesting a dose-response effect. ${ }^{20}$

Given that ofatumumab appears to result in higher infusion-related reactions (IRRs) and neutropenia compared to rituximab, it has not been pursued extensively in this setting. ${ }^{21}$

Ublituximab is a type I, glycoengineered anti-CD20 recombinant $\mathrm{mAb}$ with significantly enhanced antibodydependent, cell-mediated cytotoxicity (ADCC) compared to rituximab, in in vitro studies of CLL cell lines. ${ }^{22}$ This mAb was investigated in a Phase I/II trial in 35 patients with B-cell nonHodgkin lymphoma (NHL; FL: 12 patients) and CLL (8) with prior rituximab treatment. ${ }^{23}$ In the FL cohort, ORR was $42 \%$ (two complete responses [CRs] and three partial responses [PRs]). All patients had one or more adverse events (AEs) including 40\% IRR and at least one grade 3-4 AE reported in $49 \%$ of patients. Multiple clinical trials with ublituximab alone or in combination with other agents are ongoing.

Anti-CD20s no longer in development include Veltuzumab which is a second-generation humanized anti-CD20 $\mathrm{mAb}$ and, in vitro, functions similarly to rituximab; however, it has a framework region that differs from rituximab. ${ }^{24}$ Veltuzumab achieved an objective response rate of $44 \%$ in 82 patients with relapsed, refractory FL. ${ }^{25}$ PRO131921 is a third-generation humanized anti-CD20 antibody with increased ADCC compared to rituximab. ${ }^{26}$ In a Phase I trial in relapsed or refractory CD-20-positive indolent NHL (iNHL), a $50 \%$ response rate was achieved in rituximab-refractory FL patients. AME-133v (Mentrik Biotech, LLC., Dallas, TX, USA), an engineered humanized IgG1 mAb against CD20, demonstrated increased preclinical ADCC activity compared to rituximab. ${ }^{27}$ In a Phase I study of 23 previously treated patients with FL, there were CRs in two (9\%) and PRs in three $(13 \%)$.

\section{Obinutuzumab Mechanism of action}

Obinutuzumab (G, GA101) is a third-generation, type II, glycoengineered, humanized, anti-CD20 mAb of the IgG1 isotope. ${ }^{28} \mathrm{Fc}$ domains are engineered to improve its therapeutic activity, specifically in patients who express low-affinity Fc receptors on the immune cells. ${ }^{29}$ Many of the mechanisms of action of obinutuzumab appear distinct from those of rituximab. The different elbow-hinge amino acid sequence and $30^{\circ}$ wider elbow angle of obinutuzumab compared to a type I mAb such as rituximab results in spatial alterations of the CD20-mAb complex on B-cells. ${ }^{28,30}$ Furthermore, posttranslational glycoengineering of type II mAbs enhances binding affinity to the Fc $\gamma \mathrm{RIII}$ receptor on immune effector cells and results in increased direct cell death and ADCC/ antibody-dependent, cell-mediated phagocytosis (ADCP). ${ }^{31}$ Obinutuzumab binds CD20 in a different orientation than type I antibodies, despite identifying an overlapping epitope. It is hypothesized that the different binding topology of this class causes the two Fab arms to bind within one tetramer, whereas type I mAbs bind different tetramers with each Fab arm. Per this hypothesis, this binding type in obinutuzumab increases ADCC. ${ }^{13}$ As the result of homotypic cell aggregation, obinutuzumab induces direct non-apoptotic cell death to a greater extent and at a faster rate than either rituximab or ofatumumab. ${ }^{32-34}$ 


\section{Preclinical studies}

Obinutuzumab was found to have enhanced direct and immune effector cell-mediated cytotoxicity in whole-blood B-cell depletion and cellular assays compared with rituximab. ${ }^{28}$ Higher antitumor and B-cell-depleting activities of obinutuzumab were demonstrated in human lymphoma xenograft models and nonhuman primates. Similar preclinical findings were reported when obinutuzumab was compared to rituximab and ofatumumab in vitro and in xenograft models. ${ }^{34}$ Induction of direct cell death and ADCC were superior with obinutuzumab, whereas CDC and internalization rate were less. Moreover, obinutuzumab controlled tumor progression in rituximab-pretreated animals unlike rituximab and ofatumumab.

Obinutuzumab has demonstrated greater antitumor activity both as a single agent and in combination with cyclophosphamide (Baxter Healthcare Corporation, Deerfield, IL, USA) when compared to rituximab in xenograft models of human FL. ${ }^{35}$ The two mAbs were compared as single agents and in combination with $\mathrm{B}$, fludarabine, chlorambucil, or cyclophosphamide/vincristine (Hospira, Inc., Lake Forest, IL, USA) in xenograft models of human lymphoma and, similar to prior investigations, obinutuzumab had greater antitumor activity. ${ }^{36}$ Based on preclinical data demonstrating the potential clinical efficacy of obinutuzumab, clinical trials were designed to investigate this $\mathrm{mAb}$ in the treatment of lymphoid malignancies.

\section{Pharmacokinetics}

Pharmacokinetic data from two early phase studies of obinutuzumab monotherapy in iNHLs were utilized to determine the therapeutic dose in subsequent trials. In the Phase I GAUGUIN study, obinutuzumab was dosed every 3 weeks for eight 21-days cycles for a total of nine infusions. ${ }^{37}$ Patients received obinutuzumab in a dose-escalating algorithm (first dose/assigned dose). The first four cohorts received $50 \%$ of assigned dose in the first infusion which was followed by the full dose in the proceeding eight treatments (Dose 1-4: $50 / 100,100 / 200,200 / 400,400 / 800 \mathrm{mg}$ ). Patients in cohorts 5 and 6 received obinutuzumab at higher doses of $800 / 1200$ and $1200 / 2000 \mathrm{mg}$ as no dose-limiting toxicities were reported. The highest loading dose of $1600 \mathrm{mg}$ was given to cohort 7 on days 1 and 8 and was followed by $800 \mathrm{mg}$ in the next cycles. Cohort 7 was different as based on the preliminary pharmacokinetic analysis revealing a fast drop in antibody level after first infusion, a higher loading dose of $1600 \mathrm{mg}$ was administered at first and was followed by $800 \mathrm{mg}$ in the following cycles. Serum obinutuzumab concentrations significantly increased after doses of 400/800 mg and higher (with steady state reached after cycle 4) compared with lower dose cohorts $\left(50 / 100,100 / 200\right.$, and 200/400 mg). ${ }^{37}$ One of the differences between clinical development of obinutuzumb and rituximab is the dosing of these agents. Obinutuzumab has been mainly tested with fixed doses; however, rituximab is dosed based on body surface area (BSA). This difference raises concerns about pharmacokinetics variability in patients with low or high BSA as studies have shown that fixed rituximab dosing results in overdosing and underdosing patients with extreme BSAs, respectively. ${ }^{38}$ This subject is controversial and still being studied in obinutuzumab trials. However, the structural and functional differences of these two mAbs limit their direct pharmacological comparison. ${ }^{39}$ In the Phase II GAUGUIN trial, 40 patients with relapsed or refractory indolent B-cell lymphomas were randomized to one of two cohorts: the first group received a flat dose of 400 $\mathrm{mg}$ on days 1 and 8 of cycle 1 followed by $400 \mathrm{mg}$ on day 1 of cycles 2-8 (400/400 mg). ${ }^{40}$ Patients in the second group received obinutuzumb $1600 \mathrm{mg}$ on days 1 and 8 of cycle 1 and $800 \mathrm{mg}$ on days $2-8(1600 / 800 \mathrm{mg}){ }^{40}$ Obinutuzumab serum concentrations did not reach a steady state in the former arm, but trough levels increased to $300 \mu \mathrm{g} / \mathrm{mL}$ up to cycle 3 when the steady state was reached in the latter arm. This dose level was not associated with significantly increased AEs. To simplify the regimen for future studies, obinutuzumab $1000 \mathrm{mg}$ for all cycles (days 1, 8, and 15 in cycle 1) was evaluated and found to have a similar pharmacokinetic profile as 1600/800 mg. ${ }^{41}$ Obinutuzumab $1000 \mathrm{mg}$ on days 1,8 , and 15 of cycle 1 and day 1 of following cycles has been favored in subsequent clinical trials. It could be suspected that increased exposure to treatment using this regimen could result in superior clinical outcomes of obinutuzumab compared to rituximab; however, this has not been demonstrated in studies. Based on this regimen, patients receive a total dose of $3000 \mathrm{mg}$ during cycle 1 which is similar to $3200 \mathrm{mg}$ received in the $1600 / 800 \mathrm{mg}$ arm of GAUGIN Phase II.

\section{Obinutuzumab as a single agent}

The safety and clinical activities of obinutuzumab were evaluated in the multicenter Phase I GAUGUIN trial, which included heavily pretreated, relapsed, or refractory B-cell lymphoma patients (Table 1) ${ }^{37} \mathrm{~A}$ total of 21 patients with a median age of 64 years were enrolled and they received escalating doses of obinutuzumab monotherapy. Lymphoma subtypes included FL: 13; mantle cell lymphoma (MCL): 4; diffuse large B-cell lymphoma (DLBCL): 1; small lymphocytic (SLL): 1; lymphoplasmacytic lymphoma: 1; and 
Table I Obinutuzumab as a single agent

\begin{tabular}{|c|c|c|c|c|c|c|}
\hline Study & Phase & Diagnosis & $\begin{array}{l}\text { No. of } \\
\text { patients (FL) }\end{array}$ & Regimen & ORR (CR) in FL & Survival \\
\hline $\begin{array}{l}\text { GAUGUIN } \\
\text { Salles et a }{ }^{37}\end{array}$ & 1 & $\mathrm{R} / \mathrm{R}$ iNHL & $21(13)$ & $\begin{array}{l}50 / 100-1200 / 2000 \mathrm{mg} \\
\text { (escalating doses) }\end{array}$ & $54 \%(31 \%)$ & N/A \\
\hline GAUGUIN & II & $\mathrm{R} / \mathrm{R}$ iNHL & $18(14)$ & $400 / 400 \mathrm{mg}$ & & (Median 33.7 months PFS) \\
\hline \multirow[t]{2}{*}{ Salles et $\mathrm{al}^{40}$} & & & & & & 6 months \\
\hline & & & $22(20)$ & $1600 / 800 \mathrm{mg}$ & $50 \%$ & 11.9 months \\
\hline $\begin{array}{l}\text { GAUSS } \\
\text { Sehn et al }{ }^{42}\end{array}$ & I & $\mathrm{R} / \mathrm{R} N H L$ or $\mathrm{CLL}$ & $22(10)$ & $\begin{array}{l}200-2000 \text { mg every week } \times 4 \text {, } \\
\text { maintenance every } 3 \text { months } \\
\times 2 \text { years }\end{array}$ & $40 \%(10 \%)$ & $N / A$ \\
\hline \multirow[t]{2}{*}{$\begin{array}{l}\text { GAUSS } \\
\text { Sehn et } \mathrm{al}^{43}\end{array}$} & II & Relapsed iNHL & $88(74)$ & $\begin{array}{l}\text { G } 1000 \text { mg every week } \times 4 \text {, } \\
\text { maintenance } \times 2 \text { years or until } \\
\text { progression }\end{array}$ & $63.5 \%(38 \%)$ & 2 year PFS: $46 \%$ \\
\hline & & & $87(75)$ & $\begin{array}{l}\text { R } 375 \mathrm{mg} / \mathrm{m}^{2} \text { every week } \times 4 \text {, } \\
\text { maintenance } \times 2 \text { years or until } \\
\text { progression }\end{array}$ & $\begin{array}{l}49 \%(27 \%)(p=0.04, \\
\text { CR: } p=0.07)\end{array}$ & $\begin{array}{l}2 \text { year PFS: 50\% HR: } 0.93 \\
(95 \% \mathrm{Cl}, 0.60-1.44)\end{array}$ \\
\hline
\end{tabular}

Abbreviations: CLL, chronic lymphocytic leukemia; CR, complete response; FL, follicular lymphoma; G, obinutuzumab; HR, hazard ratio; iNHL, indolent NHLs; N/A, not applicable; NHL, non-Hodgkin lymphoma; ORR, overall response rate; PFS, progression-free survival; R, rituximab; R/R, relapsed refractory.

Waldenström macroglobulinemia: 1. Patients had received a median of five prior therapies; $95 \%$ had received prior rituximab, $45 \%$ were rituximab-refractory, and $29 \%$ had undergone a prior autologous stem cell transplant. FL patients were the only responding cohort with an ORR of $69 \%$ and $38 \%$ CRs, and no observed dose-response relationship. Obinutuzumab was well tolerated with the majority of toxicities being grade 1-2 IRRs. Considering that obinutuzumab was found to be safe and active at all dose levels, a Phase II GAUGUIN trial was conducted. ${ }^{40}$ Of the total 40 patients, histologies included 34 FL, of which $80 \%$ had intermediate or high Follicular Lymphoma International Prognostic Index (FLIPI) scores. They had received a median of three prior treatments (two rituximab treatments) and 55\% were rituximab-refractory. The ORR was $55 \%$ in the $1600 / 800 \mathrm{mg}$ group (9\% CR). In the FL patients, the ORR was 50\% (10 of 20 patients) in the 1600/800 mg group, and $21 \%$ (3 of 14 patients) in the $400 / 400 \mathrm{mg}$ group. The ORRs in rituximabrefractory patients were $8 \%$ in the $400 / 400 \mathrm{mg}$ group and $50 \%$ in the $1600 / 800 \mathrm{mg}$ group. After a median observation time of 33.7 months, the median PFS in all histologies were 11.9 and 6 months in the $1600 / 800$ and 400/400 mg groups, respectively. Obinutuzumab was well tolerated with few grade 3-4AEs which were more common in the $1600 / 800 \mathrm{mg}$ arm, and all were manageable.

GAUSS was the first Phase I study to evaluate the safety, tolerability, and pharmacokinetics of obinutuzumab induction followed by 2 years of maintenance. ${ }^{42}$ Histologies included $10 \mathrm{FL}, 5 \mathrm{CLL}, 2$ SLL, 3 DLBCL, $1 \mathrm{MCL}$, and 1 transformed lymphoma. Patients had received a median of four prior therapies including $86 \%$ with prior rituximab (68\% refractory) and three had a prior autologous stem cell transplantation (ASCT). Obinutuzumab induction was given at a dose range of 200-2000 mg once weekly for 4 weeks. After the completion of induction, eight patients who had not progressed (36\%, five with PR and three with SD and clinical benefit) continued to receive obinutuzumab as maintenance therapy. The best ORR among all histologies was $32 \%$ (7/22), with one (4.5\%) CR, and rituximab-refractory patients achieved an ORR of $15 \%$ (2/13; 1 CR, 1 PR). Four of seven responses lasted more than a year, with three patients having an ongoing response at the time of analysis (duration of response from 3 to 21.1 months). In FL patients, the ORR was 40\% (4/10), including one CR.

The Phase II of the GAUSS trial did not produce encouraging results. This study compared obinutuzumab with rituximab in a larger group of 175 patients with relapsed iNHLs (149 with FL) who required treatment. ${ }^{43}$ Patients had received a median of two prior treatments and only $10 \%$ were rituximab-refractory. They were randomized to four weekly infusions of either obinutuzumab $(1000 \mathrm{mg})$ or rituximab $\left(375 \mathrm{mg} / \mathrm{m}^{2}\right)$ followed by obinutuzumab or rituximab maintenance every 2 months for up to 2 years in patients without evidence of disease progression. At the end of induction (EOI), ORR was higher in obinutuzumab arm compared to rituximab ( $45 \%$ vs $27 \% ; p=0.01$ ), but with no difference in CR rates $(5.4 \%$ vs $4.0 \%, p=0.34$ in obinutuzumab vs rituximab arm, respectively). At a median follow-up period of 32 months, the 2-year PFS was similar at $46 \%$ and $50 \%$ for obinutuzumab and rituximab arms, respectively. A similar incidence of most toxicities was reported in both arms; however, cough and IRRs were more frequent in the obinutuzumab arm. 


\section{Obinutuzumab combination therapies \\ Early phases}

The Phase Ib multicenter GAUDI trial included 56 patients with relapsed, refractory FL to evaluate the safety and activity of induction with two doses of obinutuzumab $(400 / 400 \mathrm{mg}$ or $1600 / 800 \mathrm{mg}$ ) combined with CHOP (G-CHOP) or fludarabine and cyclophosphamide (FC). ${ }^{44}$ These patients had received one to six prior treatments. EOI ORR was higher with G-CHOP $1600 / 800 \mathrm{mg}$ at $100 \%(64 \% \mathrm{CR})$ compared to G-CHOP $400 / 400 \mathrm{mg}$ which was $93 \%$ (14\% CR). Interestingly, the ORR was higher in the 400/400 mg G-FC arm $(100 \%, 79 \% \mathrm{CR})$ compared to G-FC $1600 / 800 \mathrm{mg}(86 \%$, $21 \% \mathrm{CR}$ ). This finding was believed to reflect differences in tumor burden. All rituximab-refractory patients responded to treatment. The Phase I GAUDI study was amended to compare the safety of G-CHOP with G-B in a subset of 81 previously untreated patients with FL. ${ }^{45}$ Forty-one patients were enrolled in the G-B group and 40 in the G-CHOP group. Patients received obinutuzumab ( $1000 \mathrm{mg}$ on days 1 and 8 of cycle 1 and day 1 of subsequent cycles) plus B $\left(90 \mathrm{mg} / \mathrm{m}^{2}\right.$, four to six cycles every 4 weeks, on days 2 and 3 of cycle 1 and days 1 and 2 of subsequent cycles) or CHOP (six to eight cycles every 3 weeks). Patients in CR at the EOI were continued on maintenance with G $1000 \mathrm{mg}$ every 3 months for 2 years or until disease progression. During induction, $85 \%$ of patients in the G-B group received six cycles and $33 \%$ of patients in G-CHOP completed the maximum eight cycles (95\% received six cycles). At the end of treatment, ORR was $93 \%$ in the G-B group and $95 \%$ in the G-CHOP group. CR defined by $\mathrm{CT}$ was reported as $37 \%$ in $\mathrm{G}-\mathrm{B}$ group and $35 \%$ with G-CHOP. This rate increased at 30 months (63\% in G-B and 58\% in G-CHOP). PFS at 36 months was higher in G-B cohort (90\%) compared to G-CHOP (84\%). Two patients died due to progression of disease in G-CHOP cohort compared to one in G-B. Both regimens were well tolerated with the most commonly reported toxicities being IRR (5\% grade 3-4 in G-CHOP, $10 \%$ grade 3-4 in G-B). One patient in the G-CHOP group had to discontinue induction due to an IRR. This study demonstrated the promising efficacy and safety of obinutuzumab in the front-line treatment setting of patients with FL and instigated other combination trials of obinutuzumab with different chemotherapy agents.

The GALEN trial was a Phase Ib study of combination of obinutuzumab with lenalidomide in (Celgene Corporation, Summit, NJ, USA) 19 patients with relapsed, refractory FL. ${ }^{46}$ The median age of patients was 64 years; median number of prior treatments was two and eight patients had rituximab-refractory disease. Patients received escalating doses of lenalidomide $(10,15,20$, and $25 \mathrm{mg})$ with sequential administrations of obinutuzumab $1000 \mathrm{mg}$ for six cycles. The results of this chemotherapy-free regimen were encouraging with an ORR of $68 \%$ and a CR of 53\%; however, grade 3-4 neutropenia was reported in $40 \%$ of the patients. A $20 \mathrm{mg}$ dose of lenalidomide was recommended for subsequent trials and Phase II of this study in relapsed, refractory follicular and aggressive B-cell lymphomas is ongoing (NCT01582776). Based on studies demonstrating activity of the combination of lenalidomide and rituximab in patients with untreated iNHL, another similar study was designed. ${ }^{47,48}$ Lenalidomide (10, 15 , and $20 \mathrm{mg}$ ) was given on days $2-22$ of a 28 -day cycle and obinutuzumab was administered at a flat dose (1000 mg IV) on days $1,8,15$, and 22 of cycle 1 and day 1 of subsequent cycles. Fifteen patients were enrolled, 14 of which had FL; $21 \%$ had low, $29 \%$ had intermediate, and $50 \%$ had high FLIPI scores. The ORR was $93 \%$ and CR was $27 \%$. Twenty percent and $13 \%$ of patients in this study experienced grade 3-4 neutropenia and infections, respectively, but no patients were withdrawn from the study as a result. The combination of lenalidomide $20 \mathrm{mg}$ and obinutuzumab $1000 \mathrm{mg}$ was determined to be safe and toxicities were manageable. A Phase II of this trial is ongoing (NCT01995669).

Polatuzumab vedotin (Genentech, Inc.) is an antibody drug conjugate (ADC) which targets CD79b, a transmembrane component of the B-cell receptor. ${ }^{49}$ When combined with B and obinutuzumab in a Phase Ib/II study of relapsed/ refractory FL, it produced encouraging results with an ORR of $100 \%$ (CR 83\%). However, only six patients were enrolled in this cohort; thus, a larger sample size is needed to better evaluate this combination. The most common grade $3-4 \mathrm{AE}$ was neutropenia in $33 \%$ of the patients. Duration of responses had not been reported as the study is still ongoing. ${ }^{50}$

\section{Phase III studies}

Based on data from early phase combination therapies with obinutuzumab, a series of Phase III clinical trials were conducted (Table 2)..$^{51,52}$ The GADOLIN study was a multicenter, randomized Phase III trial that compared the efficacy and safety of obinutuzumab plus B with B monotherapy in patients with rituximab-refractory iNHL. ${ }^{51}$ Rituximabrefractory was defined as failure to respond to, or progression during or within, 6 months of the last rituximab dose Patients received either obinutuzumab $1000 \mathrm{mg}$ (days 1, 8, and 15 of cycle 1 , and day 1 of cycles 2-6) in combination with B $90 \mathrm{mg} / \mathrm{m}^{2} /$ day (days 1 and 2 of cycles $1-6$ ) or B monotherapy $\left(120 \mathrm{mg} / \mathrm{m}^{2} /\right.$ day days 1 and 2 of cycles $\left.1-6\right)$ 
Table 2 Obinutuzumab in combination therapies

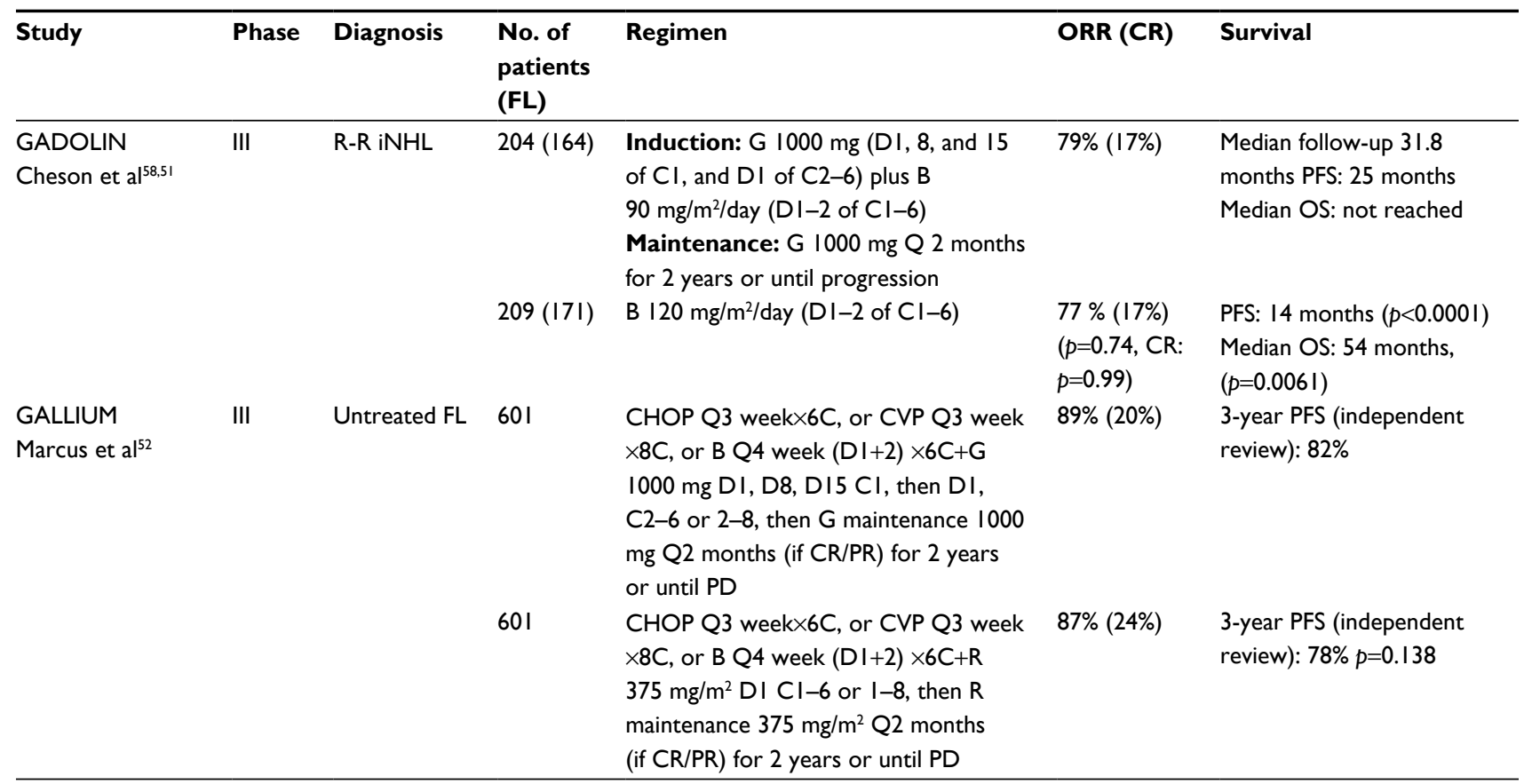

Abbreviations: B, bendamustine; C, cycle(s); CHOP, cyclophosphamide, doxorubicin, vincristine, and prednisone; CR, complete response; CVP, cyclophosphamide, vincristine, prednisone; D, day(s); FL, follicular lymphoma; G, obinutuzumab; iNHL, indolent non-Hodgkin lymphoma; ORR, overall response rate; OS overall survival; PD, progression of disease; PFS, progression-free survival; PR, partial response; R, rituximab; R-R, rituximab refractory.

in 28 days cycles. Following induction, patients in the G-B arm without evidence of progression received obinutuzumab maintenance, $1000 \mathrm{mg}$ every 2 months for 2 years or until disease progression. A higher incidence of grade $\geq 3$ AEs was reported in G-B arm compared to B arm $(72.5 \%$ vs $65.5 \%)$ which included neutropenia (34.8\% vs $27.1 \%)$ and IRRs ( $9.3 \%$ vs $3 \%)$. However, grade $\geq 3$ thrombocytopenia (10.8\% in G-B vs $15.8 \%$ in B) and anemia (7.4\% in G-B vs $10.8 \%$ in $\mathrm{B}$ ) were more common in the $\mathrm{B}$ alone group. Furthermore, serious AEs were more frequent in the G-B arm (43.6\% vs $36.9 \%)$. Safety results in FL cohort were similar to all iNHL patients.

$\mathrm{CR}$ and ORR were similar between the arms. However, in the FL cohort $(\mathrm{n}=321)$, the addition of obinutuzumab induced a longer median PFS (25 vs 14 months, $p<0.0001$, hazard ratio [HR] $0.52 ; 95 \%$ CI $0.39,0.69)$ and median OS (not reached vs 53.9 months, $p=0.0061$, HR $0.58 ; 95 \%$ CI $0.39,0.86)$. Results for all the patients with iNHL were consistent with those for FL patients. Data analysis of all enrolled patients demonstrated that the PFS was 25.8 months in the G-B arm compared to 14.1 months in the B arm (HR 0.57; 95\% CI 0.44, $p<0.0001$ ), and median OS was not reached in either arm. These data supported the approval of obinutuzumab for combination with B $\left(90 \mathrm{mg} / \mathrm{m}^{2}\right)$ in relapsed or refractory FL as it clearly improved PFS and OS. Analysis of minimal residual disease (MRD) by $\mathrm{t}(14 ; 18)$ and/or Ig variable domain allele-specific RQ-PCR in 93 biomarkerevaluable patients in FL cohort of GADOLIN trial revealed more MRD negativity mid-induction, EOI, and at the end of maintenance with G-B than with B alone ( $82 \%$ vs $43 \%$; $p<0.0001$ ), which correlated with outcome. ${ }^{53}$ Quality of life analysis also favored the G-B arm. ${ }^{54}$

The GALLIUM study is a Phase III trial in 1202 previously untreated advanced FL patients. ${ }^{52}$ Patients were randomized either to rituximab $375 \mathrm{mg} / \mathrm{m}^{2}$ (day 1 of each cycle) or obinutuzumab (1000 mg on days 1,8 , and 15 of cycle 1 and day 1 of subsequent cycles) for either six to eight 21-day cycles (CHOP and cyclophosphamide, vincristine, prednisone [CVP]) or six 28-day cycles (B). Patients with a CR or PR at the EOI received rituximab or obinutuzumab every 2 months for 2 years or until disease progression, per induction arm. Obinutuzumab and rituximab produced an ORR of $89 \%$ and $87 \%$, respectively (CR rates of $20 \%$ and $24 \%$, respectively). However, with a median follow-up of 34.5 months, a significantly higher 3-year PFS of 80 months was reported in the obinutuzumab arm, compared to 73 months in rituximab arm $(p=0.0012)$, but with no difference in overall survival $(p=0.21)$. Moreover, all toxicities were greater with $\mathrm{G}$ than $\mathrm{R}$. 
Serious AEs were more frequent in all obinutuzumabchemotherapy groups, but fatal AEs and events causing discontinuation of treatment were similar. Rates of neutropenia and febrile neutropenia were higher in G-CHOP compared to GB group ( $71 \%$ vs $30 \%$ and $11 \%$ vs $5 \%$, respectively); whereas grade 3-5 infections, fatal AEs, and secondary malignancies occurred more frequently in patients treated with GB ( $26 \%$ vs $12 \%, 6 \%$ vs $2 \%$, and $11 \%$ vs $5 \%$, respectively). Rates of grade 3-5 infections were similar in $\mathrm{G}$ and $\mathrm{R}$ arms in CHOP and CVP cohorts. ${ }^{55}$

This observation is unexplained as it has not been made in other trials. ${ }^{56,57}$

\section{Toxicities \\ Infusion-related reactions}

IRRs have been the most reported toxicity in all obinutuzumab clinical trials, occurring in up to $86 \%$ of the patients, a higher frequency than encountered with rituximab. ${ }^{37,40,42-44,51,52,59}$ These reactions, including dyspnea, tachypnea, hypotension, hypertension, flushing, tachycardia, and chills are mostly grade 1-2 and generally occur during the first infusion. IRRs can be managed by premedication with acetaminophen, antihistamine, and steroids, and with slowing or stopping the first infusion, if needed. Safety analysis for the entire treatment course in Phase II GAUSS revealed a higher rate of IRRs (all grades: $74 \%$ vs $51 \%$, grade $3-4: 11 \%$ vs $5 \%)$ and cough ( $24 \%$ vs $9 \%$ ) in the obinutuzumab arm. ${ }^{43}$ Moreover, patients on the obinutuzumab arm of GALLIUM study demonstrated a higher frequency of grade 3-4 IRRs as well (6.7\% in obinutuzumab arm vs 3.7\% in rituximab arm).${ }^{52}$ It was hypothesized that the increase in IRRs, especially during the first infusion, was due to cytokine release as it was noted that peak levels of IL-6, IL-8, IL-10, TNF- $\alpha$, and interferon-gamma were significantly higher during the first infusion of obinutuzumab compared with rituximab. Levels returned to baseline and did not increase in subsequent infusions.

\section{Hematological toxicities}

Neutropenia is one of the most common hematological AEs in patients treated with obinutuzumab. ${ }^{37,40,42-44,51,52,59}$ This toxicity can present as early onset grade 3-4 neutropenia, late or prolonged neutropenia. Delayed onset neutropenia is a known toxicity of treatment with rituximab occurring in $8 \%-25 \%$ of patients. ${ }^{60-62}$ Maturation arrest, changes in stromal-derived factor-1 (SDF01) inhibiting neutrophils from leaving the bone marrow and FCgammaR3A polymorphisms are proposed mechanisms of this rituximab complication. It is likely that similar mechanisms could play roles in obinutuzumab-related neutropenia.

In final analysis of the GAUSS study, patients with relapsed iNHL treated with obinutuzumab and rituximab developed similar rates of grade $3-4$ neutropenia ( $3 \%$ vs $6 \%$, respectively).$^{43}$ In this study, patients had an history of up to five prior regimens with rituximab which may predispose patients to recurrent episodes of neutropenia. ${ }^{60}$

In the GALLIUM study, a higher incidence of neutropenia was reported in previously untreated FL patients treated with obinutuzumab-based chemotherapy compared to rituximabbased chemotherapy ( $44 \%$ vs $38 \%)^{52}$

Studies are ongoing to investigate the incidence and etiology of neutropenia. Administration of granulocyte colonystimulating factor (G-CSF) should be considered in patients with symptomatic grade 3-4 neutropenia.

Thrombocytopenia has also been reported as a toxicity of obinutuzumab and is usually managed by dose delays or, if needed, platelet transfusion. The GALLIUM study demonstrated a low rate of grade 3-4 thrombocytopenia in both obinutuzumab and rituximab arms ( $6 \%$ vs $3 \%$, respectively). ${ }^{52}$

\section{Other adverse events}

Obinutuzumab can result in reactivation of hepatitis B virus, resulting in fulminant hepatitis and death similar to other anti-CD20-directed mAbs; hence patients should be screened prior to treatment. ${ }^{63}$ Rare cases of progressive multifocal leukoencephalopathy and disseminated enteroviral infections in patients treated with obinutuzumab have been reported. ${ }^{64,65}$

\section{Conclusion and future perspectives}

Management of FL remains challenging. Despite high response rates to standard chemoimmunotherapy, patients typically relapse. In recent years, clinical research has focused on discovering novel treatments that result in durable responses with minimum toxicity (Table 3). Obinutuzumab offers the potential for improved outcome. However, how best to integrate this antibody into treatment paradigms remains to be determined. While the GADOLIN trial demonstrated prolonged survival using obinutuzumab in rituximabrefractory FL, the GALLIUM study demonstrated benefit in the up-front setting with respect to PFS, but not OS. Thus, it remains to be seen how likely the GALLIUM regimen will be adopted in practice. A next step could be to incorporate obinutuzumab with novel agents. For example, the Phase II GALEN study as well as a similar study at MD Anderson are investigating the chemotherapy-free combination of 
Table 3 Ongoing clinical trials

\begin{tabular}{|c|c|c|c|}
\hline Identifier & Phase & Disease & Regimen \\
\hline NCT0I582776 (GALEN) & II & R/R FL & G, lenalidomide (Celgene Corporation, Summit, NJ, USA) \\
\hline NCTOI995669 & II & Relapsed iNHL & G, lenalidomide \\
\hline NCT02992522 & I & $\mathrm{R} / \mathrm{R} \mathrm{NHL}$ & G, lenalidomide, venetoclax (AbbVie Inc., North Chicago, IL, USA) \\
\hline $\begin{array}{l}\text { NCT01691898 } \\
\text { (ROMULUS) }\end{array}$ & II & $\mathrm{R} / \mathrm{R} \mathrm{NHL}$ & $\begin{array}{l}\text { G, polatuzumab vedotin (Genentech, Inc., South San Francisco, CA, USA) (CD79b antibody- } \\
\text { drug conjugate) }\end{array}$ \\
\hline NCT02877550 & I & Front-line FL & G, venetoclax (BCL-2 inhibitor) \\
\hline NCT03039। I4 & I & $\mathrm{R} / \mathrm{R} \mathrm{FL}$ & G, B, INCB050465 (Incyte, Wilmington, DE, USA) (PI3K-delta inhibitor) \\
\hline NCT02457598 & I & $\begin{array}{l}\text { R/R B-cell } \\
\text { malignancies }\end{array}$ & $\begin{array}{l}\text { Entospletinib (Chemietek, Indianapolis, IN, USA) (selective inhibitor of spleen tyrosine kinase) or } \\
\text { idelalisib (Gilead Sciences, Inc., Foster City, CA, USA) in combination with ONO/GS-4059 (Ono } \\
\text { Pharmaceutical Company, Ltd. Osaka, Japan) (selective BTK inhibitor) and G }\end{array}$ \\
\hline NCT03075696 & I & $\mathrm{R} / \mathrm{R} \mathrm{NHL}$ & G, RO7082859 (Hoffman-La Roche Ltd., Basel, Switzerland) (a novel T-cell bispecific TCB) \\
\hline NCT02624986 & $\mathrm{I} / \mathrm{II}$ & $\mathrm{R} / \mathrm{R} \mathrm{FL}$ & G, idasanutlin (Hoffman-La Roche Ltd., Basel, Switzerland) (MDM2 antagonist) \\
\hline NCT02220842 & I & $\mathrm{R} / \mathrm{R} \mathrm{FL}$ & G, atezolizumab (Genentech, Inc.) (PDL-I inhibitor) \\
\hline NCT0259697I & I & $\begin{array}{l}\text { Front-line FL } \\
\text { R/R FL }\end{array}$ & $\begin{array}{l}\text { G, B, atezolizumab } \\
\text { G, CHOP, atezolizumab }\end{array}$ \\
\hline
\end{tabular}

Abbreviations: B, bendamustine; Bcl-2, B-cell lymphoma 2; BTK, bruton tyrosine kinase; CHOP, cyclophosphamide, doxorubicin, vincristine, and prednisone; FL, follicular lymphoma; G, obinutuzumab; iNHL, indolent non-Hodgkin lymphoma; MDM2, mouse double minute 2 homolog; NHL, non-Hodgkin lymphoma; PDL-I inhibitor, programmed cell death-ligand I; R/R, relapsed and refractory; TCB, T-cell bispecific.

obinutuzumab and lenalidomide in front-line and relapsed, refractory settings. (NCT01582776, NCT01995669) These trials were designed based on the successful results of two studies with rituximab and lenalidomide doublet in untreated patients with FL demonstrating ORRs $>90 \%$ and $\mathrm{CR}$ rates $>70 \% .{ }^{47,66}$ Results of the Phase III RELEVANCE study of this combination vs chemoimmunotherapy in the front-line setting are pending (NCT01650701).

With the goal of limiting cytotoxic chemotherapy or increasing the efficacy of standard chemotherapy regimens, combination of obinutuzumab with immunomodulators and immunotherapy agents such as programmed cell death-ligand 1 (PD-1/PDL-1) inhibitors are also attractive regimens for clinical trials. Atezolizumab is a humanized $\mathrm{mAb}$ immune checkpoint inhibitor that inhibits binding of PD-L1 to PD-1 and B7-1, thereby preventing the inhibition of antitumor activity of T-cells, and has been approved in the treatment of advanced urothelial carcinoma and non-small cell lung cancer. ${ }^{67,68} \mathrm{~A}$ promising international Phase $\mathrm{Ib} / \mathrm{II}$ study of atezolizumab in combination with either obinutuzumab plus B or obinutuzumab plus CHOP in patients with FL (untreated or relapsed/refractory) is currently recruiting patients (NCT02596971). Another multicenter global trial is investigating the safety and efficacy of combining obinutuzumab with atezolizumab, without chemotherapy, in relapsed, refractory FL patients (NCT02220842).

Another challenge for obinutuzumab is the availability of anti-CD20 biosimilars and subcutaneous rituximab, which was recently approved by FDA for the treatment of patients with previously untreated and relapsed or refractory FL, DLBCL, and CLL after it was demonstrated to be non-inferior to intravenous rituximab in a randomized trial. ${ }^{69-73}$ The subcutaneous form of rituximab will significantly improve patients' convenience and use of health-care resources.

Based on recent studies showing the relationship of early relapse of FL with decreased survival, prompt initiation of more effective second-line treatment regimens with novel combinations with agents such as obinutuzumb in this group of patients should be emphasized. High-throughput screening experiment on drug combinations is being investigated as one of the novel methods to discover the most synergistic drug combinations on cell lines..$^{74} \mathrm{~A}$ US trial is being planned for patients who have failed bendamustine (Teva Pharmaceutical Industries, Petah Tikva, Israel)-based induction regimens. Patients would be randomized to one of three arms: O-CHOP, O-idelalisib, and O-lenalidomide. A variety of correlative studies would be performed to identify molecular and genetic features associated with patient outcome. More accurate clinical predictive methods could identify poor-risk patients at baseline. Use of riskadapted studies and predictive measures such as m7-FLIPI and metabolic tumor volume (MTV) prior to starting treatment could help identify patients at high risk for early progression. ${ }^{75,76}$ Furthermore, by monitoring interim MRD and positron emission tomography-computed tomography (PET-CT) scan results, patients with a likelihood of early relapse could be detected promptly which guide clinicians in initiating new treatment regimens immediately after treatment failure. ${ }^{77}$

The future landscape of treatment for FL is very encouraging. Focusing on identifying biomarkers and optimizing chemotherapy-free combinations will hopefully lead to a 
significant improvement in survival and possibly cure for patients with FL.

\section{Acknowledgment}

Lombardi Comprehensive Cancer Center received research funding from Genentech.

\section{Disclosure}

Dr. Bruce D Cheson is a consultant to Genentech. The authors report no other conflicts of interest in this work.

\section{References}

1. Shi Q, Flowers CR, Hiddemann W, et al. Thirty-month complete response as a surrogate end point in first-line follicular lymphoma therapy: an individual patient-level analysis of multiple randomized trials. J Clin Oncol. Epub 2016 Dec 28.

2. Casulo C, Byrtek M, Dawson KL, et al. Early relapse of follicular lymphoma after rituximab plus cyclophosphamide, doxorubicin, vincristine, and prednisone defines patients at high risk for death: an analysis from the National LymphoCare Study. J Clin Oncol. 2015;33(23):2516-2522.

3. Maurer MJ, Bachy E, Ghesquieres H, et al. Early event status informs subsequent outcome in newly diagnosed follicular lymphoma. $\mathrm{Am} J$ Hematol. 2016;91(11):1096-1101.

4. Hiddemann W, Kneba M, Dreyling M, et al. Frontline therapy with rituximab added to the combination of cyclophosphamide, doxorubicin, vincristine, and prednisone (CHOP) significantly improves the outcome for patients with advanced-stage follicular lymphoma compared with therapy with CHOP alone: results of a prospective randomized study of the German Low-Grade Lymphoma Study Group. Blood. 2005;106(12):3725-3732.

5. Marcus R, Imrie K, Belch A, et al. CVP chemotherapy plus rituximab compared with CVP as first-line treatment for advanced follicular lymphoma. Blood. 2005;105(4):1417-1423.

6. Marcus R, Imrie K, Solal-Celigny P, et al. Phase III study of R-CVP compared with cyclophosphamide, vincristine, and prednisone alone in patients with previously untreated advanced follicular lymphoma. J Clin Oncol. 2008;26(28):4579-4586.

7. Herold M, Haas A, Srock S, et al; East German Study Group Hematology and Oncology Study. Rituximab added to first-line mitoxantrone, chlorambucil, and prednisolone chemotherapy followed by interferon maintenance prolongs survival in patients with advanced follicular lymphoma: an East German Study Group Hematology and Oncology Study. J Clin Oncol. 2007;25(15):1986-1992.

8. Lunning MA, Vose JM. Management of indolent lymphoma: where are we now and where are we going. Blood Rev. 2012;26(6):279-288.

9. Alduaij W, Illidge TM. The future of anti-CD20 monoclonal antibodies: are we making progress? Blood. 2011;117(11):2993-3001.

10. Owen CJ, Stewart DA. Obinutuzumab for the treatment of patients with previously untreated chronic lymphocytic leukemia: overview and perspective. Ther Adv Hematol. 2015;6(4):161-170.

11. Tobinai K, Klein C, Oya N, Fingerle-Rowson G. A review of obinutuzumab (GA101), a novel type II anti-CD20 monoclonal antibody, for the treatment of patients with B-cell malignancies. Adv Ther. 2017;34(2): 324-356.

12. Maeshima AM, Taniguchi H, Fukuhara S, et al. Follow-up data of 10 patients with B-cell non-Hodgkin lymphoma with a CD20-negative phenotypic change after rituximab-containing therapy. Am J Surg Pathol. 2013;37(4):563-570.

13. Klein C, Lammens A, Schafer W, et al. Epitope interactions of monoclonal antibodies targeting CD20 and their relationship to functional properties. MAbs. 2013;5(1):22-33.

14. Cragg MS, Morgan SM, Chan HT, et al. Complement-mediated lysis by anti-CD20 mAb correlates with segregation into lipid rafts. Blood. 2003;101(3):1045-1052.
15. Cragg MS, Glennie MJ. Antibody specificity controls in vivo effector mechanisms of anti-CD20 reagents. Blood. 2004;103(7):2738-2743.

16. Beers SA, Chan CH, French RR, Cragg MS, Glennie MJ. CD20 as a target for therapeutic type I and II monoclonal antibodies. Semin Hematol. 2010;47(2):107-114.

17. Teeling JL, French RR, Cragg MS, et al. Characterization of new human CD20 monoclonal antibodies with potent cytolytic activity against nonHodgkin lymphomas. Blood. 2004;104(6):1793-1800.

18. Wierda WG, Kipps TJ, Mayer J, et al; Hx-CD20-406 Study Investigators. Ofatumumab as single-agent CD20 immunotherapy in fludarabinerefractory chronic lymphocytic leukemia. J Clin Oncol. 2010;28(10): 1749-1755.

19. Czuczman MS, Fayad L, Delwail V, et al; 405 Study Investigators. Ofatumumab monotherapy in rituximab-refractory follicular lymphoma: results from a multicenter study. Blood. 2012;119(16):3698-3704.

20. Rosenbaum CA, Pitcher B, Bartlett NL. Phase II Trial of Ofatumumab (OFA) in Previously Untreated Follicular Non-Hodgkin Lymphoma (NHL): CALGB 50,901 (Alliance). Proceedings of the 57th American Society of Hematology Annual Meeting; 2015 Dec 5-8; Orlando, Florida, US. Blood 12623(2015):2741.

21. Czuczman MS, Kahanic S, Forero A, et al. Results of a phase II study of bendamustine and ofatumumab in untreated indolent B cell nonHodgkin's lymphoma. Ann Hematol. 2015;94(4):633-641.

22. Le Garff-Tavernier M, Decocq J, de Romeuf C, et al. Analysis of CD16+ CD56dim NK cells from CLL patients: evidence supporting a therapeutic strategy with optimized anti-CD20 monoclonal antibodies. Leukemia. 2011;25(1):101-109.

23. Sawas A, Farber CM, Schreeder MT, et al. A phase $1 / 2$ trial of ublituximab, a novel anti-CD20 monoclonal antibody, in patients with B-cell non-Hodgkin lymphoma or chronic lymphocytic leukaemia previously exposed to rituximab. Br J Haematol. 2017;177(2):243-253.

24. Stein R, Qu Z, Chen S, et al. Characterization of a new humanized antiCD20 monoclonal antibody, IMMU-106, and Its use in combination with the humanized anti-CD22 antibody, epratuzumab, for the therapy of non-Hodgkin's lymphoma. Clin Cancer Res. 2004;10(8):2868-2878.

25. Morschhauser F, Leonard JP, Fayad L, et al. Humanized anti-CD20 antibody, veltuzumab, in refractory/recurrent non-Hodgkin's lymphoma: phase I/II results. J Clin Oncol. 2009;27(20):3346-3353.

26. Casulo C, Vose JM, Ho WY, et al. A phase I study of PRO131921, a novel anti-CD20 monoclonal antibody in patients with relapsed/refractory CD20+ indolent NHL: correlation between clinical responses and AUC pharmacokinetics. Clin Immunol. 2014;154(1):37-46.

27. Forero-Torres A, de Vos S, Pohlman BL, et al. Results of a phase 1 study of AME-133v (LY2469298), an Fc-engineered humanized monoclonal anti-CD20 antibody, in FcgammaRIIIa-genotyped patients with previously treated follicular lymphoma. Clin Cancer Res. 2012;18(5):1395-1403.

28. Mossner E, Brunker P, Moser S, et al. Increasing the efficacy of CD20 antibody therapy through the engineering of a new type II anti-CD20 antibody with enhanced direct and immune effector cell-mediated B-cell cytotoxicity. Blood. 2010;115(22):4393-4402.

29. Oflazoglu E, Audoly LP. Evolution of anti-CD20 monoclonal antibody therapeutics in oncology. MAbs. 2010;2(1):14-19.

30. Niederfellner G, Lammens A, Mundigl O, et al. Epitope characterization and crystal structure of GA101 provide insights into the molecular basis for type I/II distinction of CD20 antibodies. Blood. 2011;118(2):358-367.

31. Goede V, Fischer K, Humphrey K, et al. Obinutuzumab (GA101) plus chlorambucil $(\mathrm{Clb})$ or rituximab $(\mathrm{R})$ plus $\mathrm{Clb}$ versus $\mathrm{Clb}$ alone in patients with chronic lymphocytic leukemia (CLL) and preexisting medical conditions (comorbidities): final stage 1 results of the CLL11 (BO21004) phase III trial. Proceedings of the 49th American Society of Clinical Oncology Annual Meeting; 2013 May 31-June 4; Chicago, IL, US. J Clin Oncol. 2013;31 Suppl; abstr 7004.

32. Ivanov A, Beers SA, Walshe CA, et al. Monoclonal antibodies directed to CD20 and HLA-DR can elicit homotypic adhesion followed by lysosome-mediated cell death in human lymphoma and leukemia cells. J Clin Invest. 2009;119(8):2143-2159. 
33. Alduaij W, Ivanov A, Honeychurch J, et al. Novel type II anti-CD20 monoclonal antibody (GA101) evokes homotypic adhesion and actindependent, lysosome-mediated cell death in B-cell malignancies. Blood. 2011;117(17):4519-4529.

34. Herter S, Herting F, Mundigl O, et al. Preclinical activity of the type II CD20 antibody GA101 (obinutuzumab) compared with rituximab and ofatumumab in vitro and in xenograft models. Mol Cancer Ther. 2013;12(10):2031-2042.

35. Dalle S, Reslan L, Besseyre de Horts T, et al. Preclinical studies on the mechanism of action and the anti-lymphoma activity of the novel anti-CD20 antibody GA101. Mol Cancer Ther. 2011;10(1):178-185.

36. Herting F, Friess T, Bader S, et al. Enhanced anti-tumor activity of the glycoengineered type II CD20 antibody obinutuzumab (GA101) in combination with chemotherapy in xenograft models of human lymphoma. Leuk Lymphoma. 2014;55(9):2151-5160.

37. Salles G, Morschhauser F, Lamy T, et al. Phase 1 study results of the type II glycoengineered humanized anti-CD20 monoclonal antibody obinutuzumab (GA101) in B-cell lymphoma patients. Blood. 2012;119(22):5126-5132.

38. Wang DD, Zhang S, Zhao H, Men AY, Parivar K. Fixed dosing versus body size-based dosing of monoclonal antibodies in adult clinical trials. J Clin Pharmacol. 2009;49(9):1012-1024.

39. Cartron G, Watier H. Obinutuzumab: what is there to learn from clinical trials? Blood. 2017;130(5):581-589.

40. Salles GA, Morschhauser F, Solal-Celigny P, et al. Obinutuzumab (GA101) in patients with relapsed/refractory indolent non-Hodgkin lymphoma: results from the phase II GAUGUIN study. J Clin Oncol. 2013;31(23):2920-2926.

41. Cartron G, Hourcade-Potelleret F, Morschhauser F, et al. Rationale for optimal obinutuzumab/GA101 dosing regimen in B-cell non-Hodgkin lymphoma. Haematologica. 2016;101(2):226-234.

42. Sehn LH, Assouline SE, Stewart DA, et al. A phase 1 study of obinutuzumab induction followed by 2 years of maintenance in patients with relapsed CD20-positive B-cell malignancies. Blood. 2012;119(22):5118-5125.

43. Sehn LH, Goy A, Offner FC, et al. Randomized Phase II trial comparing obinutuzumab (GA101) with rituximab in patients with relapsed CD20+ indolent B-cell non-Hodgkin lymphoma: final analysis of the GAUSS Study. J Clin Oncol. 2015;33(30):3467-3474.

44. Radford J, Davies A, Cartron G, et al. Obinutuzumab (GA101) plus $\mathrm{CHOP}$ or FC in relapsed/refractory follicular lymphoma: results of the GAUDI study (BO21000). Blood. 2013;122(7):1137-1143.

45. Grigg A, Dyer MJ, Diaz MG, et al. Safety and efficacy of obinutuzumab with CHOP or bendamustine in previously untreated follicular lymphoma. Haematologica. 2017;102(4):765-772.

46. Morschhauser F, Salles G, Le Gouill S ea. A Phase Ib Study of Obinutuzumab Combined with Lenalidomide for Relapsed/Refractory Follicular B-Cell Lymphoma. Proceedings of the 56th American Society of Hematology Annual Meeting; 2015 Dec 6-9; San Francisco, CA, US. Blood. 2014;124:4458.

47. Fowler NH, Davis RE, Rawal S, et al. Safety and activity of lenalidomide and rituximab in untreated indolent lymphoma: an open-label, phase 2 trial. Lancet Oncol. 2014;15(12):1311-1318.

48. Fowler N, Pinto RM, Yoon Cheah C ea. A Phase I Study of Lenalidomide Plus a Next Generation Anti-CD20 Antibody, Obinutuzumab, in Relapsed Indolent Lymphoma. Proceedings of the 57th American Society of Hematology Annual Meeting; 2015 Dec 5-8; Orlando, Florida, US. Blood. 2015;126:2742.

49. Palanca-Wessels MC, Czuczman M, Salles G, et al. Safety and activity of the anti-CD79B antibody-drug conjugate polatuzumab vedotin in relapsed or refractory B-cell non-Hodgkin lymphoma and chronic lymphocytic leukaemia: a phase 1 study. Lancet Oncol. 2015;16(6):704-715.

50. Herrera A, Matasar M, al. ASe. Polatuzumab Vedotin Combined with Bendamustine (B) and Rituximab (R) or Obinutuzumab (G) in Patients with Relapsed or Refractory (R/R) Follicular Lymphoma (FL) or Diffuse Large B-Cell Lymphoma (DLBCL): Preliminary Results of a Phase Ib/ II Dose-Escalation Study. Proceedings of the 58th American Society of Hematology Annual Meeting; 2015 Dec 3-6; San Diego, CA, US. Blood. 2016;128:4194.
51. Cheson B, Trněný M, al. BKe. Obinutuzumab plus Bendamustine Followed by Obinutuzumab Maintenance Prolongs Overall Survival Compared with Bendamustine Alone in Patients with Rituximab-Refractory Indolent Non-Hodgkin Lymphoma: Updated Results of the GADOLIN Study. Proceedings of the 58th American Society of Hematology Annual Meeting; 2015 Dec 3-6; San Diego, CA, US. Blood. 2016;128:615.

52. Marcus RE, Davies AJ, al. AKe. Obinutuzumab-Based Induction and Maintenance Prolongs Progression-Free Survival (PFS) in Patients with Previously Untreated Follicular Lymphoma: Primary Results of the Randomized Phase 3 GALLIUM Study Proceedings of the 58th American Society of Hematology Annual Meeting; 2015 Dec 3-6; San Diego, CA, US. Blood. 2016;128:6.

53. Pott C, Belada D, Danesi N ea. Analysis of Minimal Residual Disease in Follicular Lymphoma Patients in Gadolin, a Phase III Study of Obinutuzumab Plus Bendamustine Versus Bendamustine in Relapsed/ Refractory Indolent Non-Hodgkin Lymphoma Proceedings of the 58th American Society of Hematology Annual Meeting; 2015 Dec 3-6; San Diego, CA, US. Blood. 2015;126:3978.

54. Cheson BD, Trask PC, Gribben JG, et al. Health-related quality of life and symptoms in patients with rituximab-refractory indolent nonHodgkin lymphoma treated in the phase III GADOLIN study with obinutuzumab plus bendamustine versus bendamustine alone. Ann Hematol. 2017;96(2):253-259.

55. Hiddemann W. BAM, Canales Albendea M.A. et al. Immunochemotherapy with Obinutuzumab or Rituximab in Previously Untreated Follicular Lymphoma in the Randomized Phase III GALLIUM study: Analysis by Chemotherapy Regimen. Proceedings of the 14th International Conference on Malignant Lymphoma Palazzo dei Congressi; 2017 June 14-June 17; Lugano, Switzerland.

56. Rummel MJ, Niederle N, Maschmeyer G, et al; Study group indolent Lymphomas (StiL). Bendamustine plus rituximab versus CHOP plus rituximab as first-line treatment for patients with indolent and mantlecell lymphomas: an open-label, multicentre, randomised, phase 3 noninferiority trial. Lancet. 2013;381(9873):1203-1210.

57. Flinn IW, van der Jagt R, Kahl BS, et al. Randomized trial of bendamustine-rituximab or R-CHOP/R-CVP in first-line treatment of indolent NHL or MCL: the BRIGHT study. Blood. 2014;123(19):2944-2952.

58. Sehn LH, Chua N, Mayer J, et al. Obinutuzumab plus bendamustine versus bendamustine monotherapy in patients with rituximab-refractory indolent non-Hodgkin lymphoma (GADOLIN): a randomised, controlled, openlabel, multicentre, phase 3 trial. Lancet Oncol. 2016;17(8):1081-1093.

59. Grigg A, Dyer MJ, Diaz MG, et al. Safety and efficacy of obinutuzumab with $\mathrm{CHOP}$ or bendamustine in previously untreated follicular lymphoma. Haematologica. 2017;102(4):765-772.

60. Li SC, Chen YC, Evens AM, et al. Rituximab-induced late-onset neutropenia in newly diagnosed B-cell lymphoma correlates with Fc receptor FcgammaRIIIa 158(V/F) polymorphism. Am J Hematol. 2010;85(10):810-812.

61. Tesfa D, Ajeganova S, Hagglund H, et al. Late-onset neutropenia following rituximab therapy in rheumatic diseases: association with $B$ lymphocyte depletion and infections. Arthritis Rheum. 2011;63(8):2209-2214.

62. Dunleavy K, Tay K, Wilson WH. Rituximab-associated neutropenia. Semin Hematol. 2010;47(2):180-186.

63. Law MF, Ho R, Cheung CK, et al. Prevention and management of hepatitis $B$ virus reactivation in patients with hematological malignancies treated with anticancer therapy. World J Gastroenterol. 2016;22(28):6484-6500.

64. Raisch DW, Rafi JA, Chen C, Bennett CL. Detection of cases of progressive multifocal leukoencephalopathy associated with new biologicals and targeted cancer therapies from the FDA's adverse event reporting system. Expert Opin Drug Saf. 2016;15(8):1003-1011.

65. Dendle C, Gilbertson M, Korman TM, Golder V, Morand E, Opat S. Disseminated enteroviral infection associated with obinutuzumab. Emerg Infect Dis. 2015;21(9):1661-1663.

66. Martin P, Jung SH, Johnson JL, et al. CALGB 50803 (Alliance): A phase II trial of lenalidomide plus rituximab in patients with previously untreated follicular lymphoma. Proceedings of the 50th American Society of Clinical Oncology Annual Meeting; 2014 May 30-June 3; Chicago, IL, US. J Clin Oncol. 2014;32(15 Suppl):8521. 
67. Fehrenbacher L, Spira A, Ballinger M, et al; POPLAR Study Group. Atezolizumab versus docetaxel for patients with previously treated nonsmall-cell lung cancer (POPLAR): a multicentre, open-label, phase 2 randomised controlled trial. Lancet. 2016;387(10030):1837-1846.

68. Balar AV, Galsky MD, Rosenberg JE, et al. Atezolizumab as first-line treatment in cisplatin-ineligible patients with locally advanced and metastatic urothelial carcinoma: a single-arm, multicentre, phase 2 trial. Lancet. 2017;389(10064):67-76.

69. Jurczak W, Moreira I, Kanakasetty GB, et al. Rituximab biosimilar and reference rituximab in patients with previously untreated advanced follicular lymphoma (ASSIST-FL): primary results from a confirmatory phase 3, double-blind, randomised, controlled study. Lancet Haematol. 2017;4(8):e350-e361.

70. Kim WS, Buske C, Ogura M, et al. Efficacy, pharmacokinetics, and safety of the biosimilar CT-P10 compared with rituximab in patients with previously untreated advanced-stage follicular lymphoma: a randomised, double-blind, parallel-group, non-inferiority phase 3 trial. Lancet Haematol. 2017;4(8):e362-e373.

71. Davies A, Merli F, Mihaljevic B, et al. Pharmacokinetics and safety of subcutaneous rituximab in follicular lymphoma (SABRINA): stage 1 analysis of a randomised phase 3 study. Lancet Oncol. 2014;15(3):343-352.
72. Davies A, Merli F, Mihaljevic B, et al. Efficacy and safety of subcutaneous rituximab versus intravenous rituximab for first-line treatment of follicular lymphoma (SABRINA): a randomised, open-label, phase 3 trial. Lancet Haematol. 2017;4(6):e272-e282.

73. RITUXAN HYCELA ${ }^{\mathrm{TM}}$ (rituximab and hyaluronidase human) [package insert]. Injection, for subcutaneous use, Genentech, Inc; San Francisco, CA, USA. Initial U.S. Approval: 2017.

74. Younes A, Ansell S, Fowler N, et al. The landscape of new drugs in lymphoma. Nat Rev Clin Oncol. 2017;14(6):335-346.

75. Pastore A, Jurinovic V, Kridel R, et al. Integration of gene mutations in risk prognostication for patients receiving first-line immunochemotherapy for follicular lymphoma: a retrospective analysis of a prospective clinical trial and validation in a population-based registry. Lancet Oncol. 2015;16(9):1111-1122.

76. Meignan M, Cottereau AS, Versari A, et al. Baseline metabolic tumor volume predicts outcome in high-tumor-burden follicular lymphoma: a pooled analysis of three multicenter studies. J Clin Oncol. Epub 2016 Aug 22.

77. Armand P, Oki Y, Neuberg DS, et al. Detection of circulating tumour DNA in patients with aggressive B-cell non-Hodgkin lymphoma. $\mathrm{Br} J$ Haematol. 2013;163(1):123-126.
Blood and Lymphatic Cancer: Targets and Therapy

\section{Publish your work in this journal}

Blood and Lymphatic Cancer: Targets and Therapy is an international, peer-reviewed, open access journal focusing on blood and lymphatic cancer research, identification of therapeutic targets and the optimal use of preventative and integrated treatment interventions to achieve improved outcomes, enhanced survival and quality of life for the

\section{Dovepress}

cancer patient. The manuscript management system is completely online and includes a very quick and fair peer-review system. Visit http://www.dovepress.com/testimonials.php to read real quotes from published authors.

Submit your manuscript here: https://www.dovepress.com/breast-cancer---targets-and-therapy-journal 